\title{
Medidas para Prevenir el COVID-19 en el Consultorio Dental
}

\author{
Measures to Prevent COVID-19 in the Dental Office
}

\author{
Britto E. Falcón-Guerrero¹ \& Guido S. Falcón-Pasapera²
}

FALCÓN-GUERRERO, B. E. \& FALCÓN-PASAPERA, G. S. Medidas para prevenir el COVID-19 en el consultorio dental. Int. J. Odontostomat., 14(4):468-473, 2020.

RESUMEN: El 11 de marzo del 2020, la OMS reconoció al COVID-19 como pandemia mundial. El coronavirus (COVID-19) ha desafiado a los sistemas de salud y demanda una rápida reacción de respuesta, ante el aumento de infectados. El ámbito del trabajo odontológico, está sujeto a un riesgo significativo de contaminación cruzada y difusión de esta enfermedad, siendo muy importante las estrictas medidas de protección. El objetivo de esta revisión es informar sobre las medidas que se deben realizar durante los tratamientos dentro del consultorio dental para prevenir la propagación del COVID-19.

PALABRAS CLAVE: Coronavirus, COVID-19, prevención, infección, odontología.

\section{INTRODUCCIÓN}

El COVID-19, es una enfermedad causada por un nuevo coronavirus, que tiene una morbilidad alta, específicamente en los ancianos y en las poblaciones comórbidas (Basile et al., 2020). Este es un bcoronavirus, que está envuelto en un virus de ARN de sentido positivo no segmentado (sarbecovirus subgénero, subfamilia de Orthocoronavirinae); según la secuenciación del genoma del virus y el análisis evolutivo, se sospecha que el murciélago es el huésped natural del origen del virus, y podría transmitirse del murciélago a través de huéspedes intermedios desconocidos hasta llegar a infectar a los humanos (Chen et al., 2020a; Guo et al., 2020; Feng He et al., 2020).

El COVID-19, causada por el síndrome respiratorio agudo severo coronavirus-2 (SARS-CoV-2) surgió en diciembre de 2019 en Wuhan, China, se ha extendido a más de 113 países; y con 118,326 infectados y 4,292 fallecidos al 11 de marzo 2020, este mismo día, la Organización Mundial de la Salud (OMS) anunció al COVID-19 como una pandemia mundial. (Wan et al., 2020a; Sun et al., 2020; Ye et al., 2020; Smith et al., 2020).
Dentro de la sintomatología, la fiebre es el síntoma más común, luego la tos, disnea y fatiga/mialgia; y síntomas menos comunes, donde destacan el dolor de cabeza y confusión, náuseas o vómitos, hemoptisis y diarrea. Se observó rinorrea y esputo en solo el $4 \%$, dolor de garganta en el $5 \%$ y faringoalgia en el $7,4 \%$ de los pacientes con información clínica relevante ( Lai et al., 2020a; Guan et al., 2020; Hui et al., 2019; Chen et al., 2020b; Huang et al., 2020; Ding et al., 2020).

La estimación actual del período de incubación medio para el COVID-19 es de 6,4 días, que oscila entre 2,1 y 14,1 días, y podría ser de 0 hasta 24 días en casos excepcionales, con posible transmisión asintomática. (Guan et al; Repici et al., 2020) y, Backer et al. (2020); mencionaron un período de incubación promedio de COVID-19 de 6,4 días que varía de 2,1 a 11,1 días.

Inicialmente, se pensó que la transmisión era de animal a humano. Pero el aumento de casos ha demostrado que la transmisión principal es de humano a humano, a través de aspirados respiratorios, de

\footnotetext{
${ }^{1}$ Facultad de odontología, Universidad Latinoamericana CIMA, Tacna, Perú.

${ }^{2}$ Facultad de odontología, Universidad Peruana Cayetano Heredia, Lima, Perú.
} 
gotitas, heces, y por aerosoles (Lai et al., 2020b). Ante la ausencia de vacunas o medicamentos antivirales para el tratamiento de COVID-19, se han reforzado las medidas de higiene y el distanciamiento social, así como restricciones de movimiento (Mizumoto et al., 2020).

El trabajo en el consultorio dental demanda un alto riesgo de infección cruzada, siendo necesario protocolos estrictos y efectivos, para el control de infecciones respiratorias transmisibles como el COVID19. (Meng et al., 2020; Sabino-Silva et al., 2020).

Esta revisión tiene como objetivo informar sobre las medidas que se deben realizar durante los tratamientos dentro del consultorio dental.

\section{RIESGOS EN EL CONSULTORIO DENTAL}

El papel de los profesionales dentales en la prevención de la transmisión de COVID-19 es de importancia crítica, si bien toda la atención odontológica de rutina se ha suspendido, la necesidad de la atención de urgencias con personal provistos de equipo de protección adecuado tiene prioridad para reducir el riesgo de exposición a enfermedades infecciosas, sobre todo en pacientes durante el período no sintomático y que llevan sin problema sus actividades habituales; lo que contribuye a la propagación de la infección, ya sea a los que viven en el mismo hogar; o por encontrarse a menos de 2 metros de una persona sana por más de 15 minutos (Repici et al.; Coulthard, 2020; Hu et al., 2020; Wang et al., 2020a). Se debe considerar que este nuevo coronavirus es muy infeccioso y transmisible; siendo necesario que el personal de salud debe usar siempre medíos de protección personal, cuando examinen casos sospechosos. (Han et al., 2020; Lu et al., 2020; Li et al., 2020a).

Dentro del consultorio dental estamos expuestos a muchos microorganismos por la presencia de aerosoles que constituyen una fuente importante de emisión de microorganismos, siendo imprescindible cumplir con todas las normas de bioseguridad para ayudar a prevenir la transmisión del COVID-19 dentro del consultorio dental; por lo tanto, la disponibilidad y el uso apropiado de equipo de protección personal, son cruciales para proteger la salud de los profesionales de la salud. (Bustamante et al., 2014; Zhang et al., 2020; Wang et al., 2020a).
Para realizar un análisis exhaustivo del paciente, se debe considerar características epidemio-lógicas y clínicas:

\section{A) Historial epidemiológico:}

1. Un historial de viaje en zonas con casos de COVID19 en los últimos 14 días; (

2. Contacto con personas infectadas;

3. Contacto con pacientes con fiebre o síntomas respiratorios en los últimos 14 días;

4. Contacto con un grupo de casos confirmados $(\geq 2$ casos con fiebre y/o síntomas respiratorios ocurrieron dentro de 2 semanas en el hogar, la oficina, la escuela, etc.).

\section{B) Manifestaciones clínicas:}

1. Fiebre y/o síntomas respiratorios;

2. Con características radiográficas de COVID-19;

3. Recuentos totales de glóbulos blancos que muestran recuento de linfocitos normal, disminuido o reducido en la etapa de inicio temprano.

Esta evaluación permite clasificar a los pacientes como de bajo, intermedio y alto riesgo, lo que se traduce en diferentes modalidades de precauciones de control de la infección. (Repici et al.; Han \& Yuang)

Para reducir los contagios en Asia se ha realizado lo siguiente:

1. Reducir la llegada de nuevos casos a la comunidad (restricciones de viaje).

2. Prevenir específicamente la posible transmisión entre casos conocidos y la población local (cuarentenas).

3. Se suprimen la transmisión silenciosa en la comunidad al reducir el contacto entre los individuos (autoaislamiento, distanciamiento social, mayor higiene) (Wu et al., 2020).

La OMS y los CDC han publicado una guía sobre hallazgos clínicos y epidemiológicos clave que sugieren una infección por COVID-19. (Tabla I) (Sohrabi et al., 2020).

Por lo que, se recomiendan medidas básicas de higiene, incluido el lavado frecuente de manos y el uso de equipos de protección personal, como máscaras faciales. (Sohrabi et al.).

Recientemente, se identificó el COVID-19 en la saliva de pacientes infectados, por lo tanto, la transmisión se da a través del contacto con gotas al hablar, toser, estornudar (actividades respiratorias hu- 
Tabla I. Comparación de los criterios de diagnóstico de los CDC versus la OMS en función de los síntomas y los viajes.

\begin{tabular}{|c|c|c|}
\hline & CDC & OMS \\
\hline $\begin{array}{l}\text { Características } \\
\text { clínicas }\end{array}$ & $\begin{array}{ll}\text { - } & \text { Fiebre. } \\
\text { Infección del } & \text { tracto } \\
\text { respiratorio } & \text { inferior } \\
\text { (posiblemente requiriendo } & \\
\text { hospitalización). } & \end{array}$ & $\begin{array}{ll}\text { - } & \text { Infección respiratoria aguda }(\mathbb{R A A}) . \\
\text { - } & \text { Fiebre o te mperatura medida } \geq 38 \mathrm{C} . \\
\text { - } & \text { Inicio en los últimos } \sim 10 \text { días. } \\
\text { - } & \text { Requiere hospitalización. }\end{array}$ \\
\hline $\begin{array}{l}\text { Riesgo } \\
\text { epidemiológico }\end{array}$ & $\begin{array}{l}\text { - Historia de viaje desde la } \\
\text { provincia de Hubei. } \\
\text { Historia de viajes desde } \\
\text { China continental. } \\
\text { Contacto cercano con } \\
\text { pacientes confirmados por } \\
\text { laboratorio con COVID-19 } \\
\text { dentro de los } 14 \text { días } \\
\text { posteriores al inicio de los } \\
\text { síntomas. }\end{array}$ & $\begin{array}{l}\text { - Historia del viaje desde la provincia de Hubei. } \\
\text { Trabajadores de la salud que han trabajado en un entorn } \\
\text { donde se atiende a pacientes con IRA. } \\
\text { El curso clínioo inesperado sigue a pesar del tratamientc } \\
\text { incluido el deterioro rápido. } \\
\text { - Contacto cercano (dentro de } 2 \text { metros durante más de } 1 \\
\text { minutos) con infección confirmada por COVID-19. } \\
\text { - Presente en centros de salud y h ospitales en paíse } \\
\text { donde se ha informado COVID-19. } \\
\text { Todo lo anterior ocurre dentro de los } 14 \text { días anteriores } \\
\text { inicio de los síntomas. }\end{array}$ \\
\hline
\end{tabular}

Tabla II. tabla de orientación de categorías de tratamientos dentales y la variedad que se pueden proporcionar al paciente durante la pandemia de COVID-19. (* Generalmente administrado por cirujanos orales y maxilofaciales.

\begin{tabular}{|c|c|c|c|c|}
\hline \multicolumn{5}{|c|}{ CATEGORİAS DE TRATAMIENTOS DENTALES } \\
\hline $\mathrm{A}$ & $\mathrm{B}$ & $\mathrm{C}$ & $\mathrm{D}$ & $E$ \\
\hline Emergencia & $\begin{array}{l}\text { Condiciones urgentes que } \\
\text { pueden manejarse con } \\
\text { procedimientos } \\
\text { mínimamente invasivos y } \\
\text { sin generación de } \\
\text { aerosoles. }\end{array}$ & $\begin{array}{l}\text { Condic iones urgentes que } \\
\text { deben manejarse con } \\
\text { procedimientos invasivos y / } \\
\text { o generadores de aerosoles }\end{array}$ & No urgente & Electivo \\
\hline $\begin{array}{l}\text { Infección bacteriana difusa } \\
\text { de tejidos blandos con } \\
\text { inflamación intraoral o } \\
\text { extraoral que puede } \\
\text { comprometer las vías } \\
\text { respiratorias del paciente. }\end{array}$ & $\begin{array}{l}\text { Dolor dental severo }(7 \leq) \text { de } \\
\text { un diente vital fracturado } \\
\text { que puede manejarse sin } \\
\text { generación de aerosol. ** }\end{array}$ & $\begin{array}{l}\text { Dolor dental severo }(7 \leq) \text { de } \\
\text { un diente vital fracturado que } \\
\text { debe manejarse con } \\
\text { proce dimientos generadores } \\
\text { de aerosol. ** }\end{array}$ & $\begin{array}{l}\text { Restauración } \\
\text { fracturada o } \\
\text { defectuosa } \\
\text { asintomática. }\end{array}$ & $\begin{array}{l}\text { Procedimientos } \\
\text { dentales estéticos. }\end{array}$ \\
\hline $\begin{array}{l}\text { Sangrado postoperatorio } \\
\text { no controlado. * }\end{array}$ & $\begin{array}{l}\text { Trauma dental con } \\
\text { avulsión / luxación que se } \\
\text { puede manejar } \\
\text { mínimamente sin } \\
\text { generación de aerosol. }\end{array}$ & $\begin{array}{l}\text { Trauma dental con avulsión / } \\
\text { luxación que se puede } \\
\text { manejar mínimamente sin } \\
\text { generación de aerosol. }\end{array}$ & $\begin{array}{l}\text { Prótesis fija } \\
\text { fracturada } \\
\text { asintomática o } \\
\text { defectuosa. }\end{array}$ & $\begin{array}{l}\text { Tratamiento } \\
\text { restaurador de } \\
\text { dientes } \\
\text { asintomáticos. }\end{array}$ \\
\hline & $\begin{array}{l}\text { Oste ítis postoperatoria } \\
\text { quirúrgica o cavidad seca } \\
\text { que puede manejarse sin } \\
\text { generación de aerosol. * }\end{array}$ & $\begin{array}{l}\text { Limpieza de prótesis fija } \\
\text { deshuesada y cementación } \\
\text { temporal. }\end{array}$ & $\begin{array}{l}\text { Aparato de } \\
\text { ortodoncia } \\
\text { fracturado o } \\
\text { defectuoso } \\
\text { asintomático. }\end{array}$ & $\begin{array}{l}\text { Extracción de } \\
\text { dientes } \\
\text { asintomáticos. }\end{array}$ \\
\hline & $\begin{array}{l}\text { Pericoronitis o dolor del } \\
\text { tercer molar que puede } \\
\text { manejarse sin generación } \\
\text { de aerosol. }\end{array}$ & $\begin{array}{l}\text { Ajustes de prótesis } \\
\text { removibles para pacientes } \\
\text { con radiación / oncología. }\end{array}$ & $\begin{array}{l}\text { Enfermedad } \\
\text { periodontal crónica. }\end{array}$ & $\begin{array}{l}\text { Procedimientos de } \\
\text { ortodoncia distintos } \\
\text { de los de la } \\
\text { categoría B / C. }\end{array}$ \\
\hline & $\begin{array}{l}\text { Fracturas maxilofaciales } \\
\text { estables que no requieren } \\
\text { intervención. * }\end{array}$ & $\begin{array}{l}\text { Prótesis fija fracturada o } \\
\text { defectuosa que causa } \\
\text { lesiones en los tejidos } \\
\text { blandos. }\end{array}$ & & $\begin{array}{l}\text { Limpieza dental de } \\
\text { rutina y terapias } \\
\text { preventivas. }\end{array}$ \\
\hline & $\begin{array}{l}\text { Absceso dental / } \\
\text { periodontal localizado que } \\
\text { puede manejarse sin } \\
\text { generación de aerosol. }\end{array}$ & $\begin{array}{l}\text { Enfermedad periodontal } \\
\text { aguda. }\end{array}$ & & $\begin{array}{l}\text { Reemplazo de } \\
\text { diente / dientes } \\
\text { faltantes con } \\
\text { prótesis fija o } \\
\text { removible. }\end{array}$ \\
\hline & $\begin{array}{l}\text { Aparato de ortodoncia fijo } \\
\text { fracturado o defectuoso }\end{array}$ & & & $\begin{array}{l}\text { Cirugía de } \\
\text { implantes dentales. }\end{array}$ \\
\hline & $\begin{array}{l}\text { que causa laceración de } \\
\text { tejidos blandos. }\end{array}$ & & & \\
\hline
\end{tabular}

** La evaluación del dolor se lleva a cabo utilizando la Herramienta universal de evaluación del dolor (UPA). 
manas) y aerosoles generados durante los procedimientos clínicos. Por ende, los consultorios dentales invariablemente conllevan el riesgo de infección COVID-19 debido a la especificidad de sus procedimientos, que implica la comunicación cara a cara con los pacientes, la exposición frecuente a saliva y sangre, y el manejo de instrumentos punzo-cortantes. (Meng et al.; Martelli-Júnior et al., 2020).

En tal razón, previo a realizar los tratamientos dentales, se debe agrupar a los pacientes en base a cinco categorías: A. Manejo de emergencia de afecciones potencialmente mortales. B. Condiciones urgentes que pueden manejarse con procedimientos mínimamente invasivos y sin generación de aerosoles. C. Condiciones urgentes que deben manejarse con procedimientos invasivos y / o generadores de aerosoles. D. Procedimientos no urgentes. E. Procedimientos electivos (Tabla II) (Alharbi et al., 2020).

\section{CONSIDERACIONES PARA LOS TRATAMIENTOS DENTALES}

Durante la realización de los tratamientos dentales se debe controlar la producción de aerosoles, que pueden ocasionar la transmisión de enfermedades en un ambiente relativamente cerrado. En tal razón se debe tener en cuenta los siguientes aspectos:

1. De ser posible, se debe evitar los tratamientos dentales.

2. Se debe evitar las radiografías periapicales, para reducir la salivación excesiva y el reflejo nauseoso, se deben utilizar de preferencia las radiografías extraorales.

3. El uso de un enjuague bucal debe ser con elementos oxidativos, como la yodo povidona al 0,23 $\%$ o peróxido de hidrógeno al $1 \%$, durante al menos 15 segundos antes del procedimiento puede reducir la carga viral en la saliva del paciente; tener en cuenta que los enjuagues con clorhexidina, parecen no ser eficaces para matar al virus.

4. Deben usarse instrumentos y dispositivos desechables y de un solo uso siempre que sea posible para reducir los riesgos de infección cruzada. 5. El dique de goma debe usarse siempre que sea posible ya que esto reducirá significativamente la propagación de microorganismos.

6. El tratamiento dental debe ser lo menos invasivo posible.
7. Los procedimientos de generación de aerosoles deben evitarse siempre que sea posible.

8. Siempre que se requiera un tratamiento farmacológico del dolor, se debe evitar el ibuprofeno en casos sospechosos y confirmados de COVID-19.

9. Los pacientes con signos y síntomas de gripe o resfrío, deben usar mascarillas en la sala de espera para reducir la descarga de gotitas y aerosoles patógenos.

10. Evitar el uso de la jeringa triple, tanto para secar y lavar intraoralmente, en su forma de spray; preferir el secado con algodón de ser posible.

11. Usar pieza de mano con válvulas de retracción, para evitar aspirar y expulsar desechos y fluidos durante los procedimientos dentales.

12. Use de una potente bomba de aspiración quirúrgica de aire / agua cerca del diente y una segunda succión cerca de la nariz para evitar la difusión de gotas de aerosol y saliva (Meng et al.; Alharbi et al.; Yu et al., 2020; Peng et al., 2020; Prati et al., 2020).

Los ambientes dentales tienen una gran cantidad de superficies potencialmente contaminables, como el sillón dental, sus manijas, la escupidera y los instrumentos dentales, teniendo en cuenta que el virus COVID-19 puede persistir en superficies hasta por 72 horas, es importante que se realice la descontaminación de todas las superficies de contacto dentro del consultorio entre paciente y paciente. Las superficies limpias deben limpiarse con agua y detergente. Para la desinfección superficial usar hipoclorito de sodio al $0,1 \%$ o etanol al 70 $\%$ o peróxido de hidrógeno al 0,5\%. (Alharbi et al.; Prati et al.; Mallineni et al., 2020) Otra alternativa, para el sillón dental es utilizar un paño suave que este humedecido con cloro de $2000 \mathrm{mg} / \mathrm{L}$ o etanol al $75 \%$. Cuando se use el cloro, los excesos y sobrantes deben limpiarse con un paño suave después de la desinfección, y cuando se usa alcohol al $75 \%$ se debe dejar que seque naturalmente (An et al., 2020).

Otro punto importante dentro de la ecuación para prevenir la contaminación en el consultorio dental, son las medidas de protección personal, que deben segur tanto el personal de salud como los pacientes:

1. Lávese las manos cuando llegue a casa o al trabajo, con agua y jabón durante al menos 20 segundos. 
2. Use gel desinfectante a base de alcohol si no hay agua y jabón disponibles; las fórmulas con al menos un $60 \%$ de etanol y/o con un $75 \%$ de alcohol, desnaturalizan las proteínas e inactivan al virus.

3. El uso de guantes no reemplaza el lavado de manos, y se requiere lavar o desinfectar las manos después de quitarse los guantes.

4. Utilizar siempre lentes de protección ocular y mascaras faciales para proteger las membranas mucosas de los ojos, la nariz y la boca.

5. Usar bata de aislamiento, guantes, gorro y mascarilla quirúrgica N95 (durante los procedimientos que generen aerosoles), para evitar el contacto con la saliva, la sangre u otros fluidos corporales del paciente.

6. Trate de evitar el contacto cercano con personas que no están bien.

7. No toque sus ojos, nariz o boca si sus manos no están limpias.

8. Si tiene fiebre, tos y dificultad para respirar, busque atención médica temprana. (Sabino-Silva et al.; Peng et al.; Prati et al.; Li et al., 2020b).

\section{CONCLUSIÓN}

El riesgo de contaminación dentro del consultorio dental es alto y ante cualquier urgencia del paciente, siempre se debe considerar la prioridad del tratamiento a realizar; considerando todas las medidas para prevenir la transmisión del COVID-19. Siendo necesario redefinir el papel del odontólogo en la prevención y monitoreo de estas infecciones virales.

FALCÓN-GUERRERO, B. E. \& FALCÓN-PASAPERA, G. S. Measures to prevent COVID-19 in the dental office. Int. J. Odontostomat., 14(4):468-473, 2020.

ABSTRACT: On March 11, 2020, the WHO recognized COVID-19 as a global pandemic. The coronavirus (COVID-19) has challenged health systems and demands a quick response reaction, due to the increase in infected people. The field of dental work is subject to a significant risk of cross contamination and spread of this disease, with strict protection measures being very important. The aim of this review is to inform about the measures that must be carried out during treatments in the dental office to prevent the spread of COVID-19.

KEY WORDS: coronavirus, COVID-19, control, prevention, infection, dentistry.

\section{REFERENCIAS BIBLIOGRÁFICAS}

Alharbi, A.; Alharbi, S. \& Alqaidi, S. Guidelines for dental care provision during the COVID-19 pandemic. Saudi Dent. J., 32(4):181-6, 2020.

An, P.; Ye, Y.; Chen, M.; Chen, Y.; Fan, W. \& Wang, Y. Management strategy of novel coronavirus (COVID-19) pneumonia in the radiology department: a Chinese experience. Diagn. Interv. Radiol., 26(3):200-3, 2020.

Backer, J.A.; Klinkenberg, D. \& Wallinga, J. Incubation period of 2019 novel coronavirus (2019-nCoV) infections among travellers from Wuhan, China, 20-28 January 2020. Euro. Surveill., 25(5):2020. 2000062. doi:10.2807/15607917.ES.2020. 25.5.2000062.

Basile, C.; Combe, C.; Pizzarelli, F.; Covic, A.; Davenport, A.; Kanbay, M.; Kirmizis, D.; Schneditz, D.; van der Sande, F. \& Mitra, S. Recommendations for the prevention, mitigation and containment of the emerging SARS-CoV-2 (COVID-19) pandemic in haemodialysis centres. Nephrol. Dial. Transplant., 35(5):737-41, 2020.

Bustamante, A. M. F.; Herrera, M. J.; Ferreira, A. R. \& Riquelme, S. D. Analysis of bacterial contamination produced by aerosols in dental clinic environments. Int. J. Odontostomat., 8(1):99105, 2014

Chen, Y.; Liu, Q. \& Guo, D. Emerging coronaviruses: Genome structure, replication, and pathogenesis. J. Med. Virol., 92(4):418-23, 2020.

Chen, N.; Zhou, M.; Dong, X.; Qu, J.; Gong, F.; Han, Y.; Qu, Y.; Wang, J.; Liu, Y.; Wei, Y.; et al. Epidemiological and clinical characteristics of 99 cases of 2019 novel coronavirus pneumonia in Wuhan, China: a descriptive study. Lancet, 395(10223):507-13, 2020.

Coulthard, P. Dentistry and Coronavirus (COVID-19) - Moral Decision-Making. Br. Dent. J., 228(7):503-5, 2020.

Ding, Q.; Lu, P.; Fan, Y.; Xia, Y. \& Liu, M. The clinical characteristics of pneumonia patients coinfected with 2019 novel coronavirus and influenza virus in Wuhan, China. J. Med. Virol., 2020. DOI: https://www.doi.org/10.1002/jmv.25781

Feng He, F.; Deng, Y. \& Li, W. Coronavirus Disease 2019 (COVID19): What we know?. J. Med. Virol., 2020. doi:10.1002/ jmv. 25766

Guan, W. J.; Ni, Z. Y.; Hu, Y.; Liang, W. H.; Ou, C. Q.; He, J. X.; Liu, L.; Shan, H.; Lei, C. L.; Hui, D. S.C.; et al. Clinical characteristics of Coronavirus disease 2019 in China. N. Engl. J. Med., 382:1708-20, 2020.

Guo, Y.R.; Cao, Q.D.; Hong, Z.S.; Tan, Y.Y.; Chen, S.D.; Jin, H.J. et al. The origin, transmission and clinical therapies on Coronavirus disease 2019 (COVID-19) outbreak - An update on the status. Mil. Med. Res., 7(1):11, 2020.

Han, Y. \& Yang, H. The transmission and diagnosis of 2019 novel coronavirus infection disease (COVID-19): A Chinese perspective. J. Med. Virol., 2020. DOI: https://www.doi.org/ 10.1002/jmv. 25749

Hu, Z.; Song, C.; Xu, C.; Jin, G.; Chen, Y.; Xu, X.; Ma, H.; Che, W.; Lin, Y.; Zheng, Y.; et al. Clinical characteristics of 24 asymptomatic infections with COVID-19 screened among close contacts in Nanjing, China. Sci. China Life Sci., 63(5):706-11, 2020.

Huang, C.; Wang, Y.; Li, X.; Ren, L.; Zhao, J.; Hu, Y.; Zhang, L.; Fan, G.; Xu, J.; Gu, X.; et al. Clinical features of patients infected with 2019 novel Coronavirus in Wuhan, China. Lancet, 395(10223):497-506, 2020.

Hui, D. S. C. \& Zumla, A. Severe acute respiratory syndrome: historical, epidemiologic, and clinical features. Infect. Dis. Clin. North Am., 33(4):869-89, 2019. 
Lai, C. C.; Liu, Y. H.; Wang, C. Y.; Wang, Y. H.; Hsueh, S. C.; Yen, M.Y.; Ko, W. C. \& Hsueh, P. R. Asymptomatic carrier state, acute respiratory disease, and pneumonia due to severe acute respiratory syndrome Coronavirus 2 (SARS-CoV-2): Facts and myths. J. Microbiol. Immunol. Infect., 2020. DOI: https:// www.doi.org/10.1016/j.jmii.2020.02.012

Lai, C. C.; Shih, T. P.; Ko, W. C.; Tang, H. J. \& Hsueh, P. R. Severe acute respiratory syndrome coronavirus 2 (SARS-CoV-2) and Coronavirus Disease-2019 (COVID-19): The epidemic and the challenges. Int. J. Antimicrob. Agents, 55(3):105924, 2020.

Li, Y.; Guo, F. F.; Cao, Y.; Li, L. F. \& Guo, Y. J. Insight Into COVID2019 for pediatricians. Pediatr. Pulmonol., 55(5):E1-E4, 2020.

Li, Z.Y. \& Meng, L.Y. The prevention and control of a new Coronavirus infection in Department of Stomatology. Zhonghua Kou Qiang Yi Xue Za Zhi, 55(0):E001, 2020.

Lu, C. W.; Liu, X. F. \& Jia, Z. F. 2019-nCoV transmission through the ocular surface must not be ignored. Lancet, 395(10224):e39, 2020.

Mallineni, S. K.; Innes, N. P.; Raggio, D. P.; Araujo, M. P.; Robertson, M. D. \& Jayaraman, J. Coronavirus disease (COVID-19): Characteristics in children and considerations for dentists providing their care. Int. J. Paediatr. Dent., 2020. DOI: https://www.doi.org/10.1111/ipd.12653

Martelli-Júnior, H.; Machado, R. A.; Martelli, D. R. B. \& Coletta, R. D. Dental journals and Coronavirus Disease (COVID-19): A current view. Oral Oncol., 104664, 2020.

Meng, L.; Hua, F. \& Bian, Z. Coronavirus Disease 2019 (COVID19): emerging and future challenges for dental and oral medicine. J. Dent. Res., 99(5):481-7, 2020.

Mizumoto, K. \& Chowell, G. Estimating risk for death from Coronavirus Disease, China, January-February 2020. Emerg. Infect. Dis., 26(6):1251-6, 2020.

Peng, X.; Xu, X.; Li, Y.; Cheng, L.; Zhou, X. \& Ren, B. Rotas de transmissão do 2019-nCoV e controles na prática odontológica. Int. J. Oral Sci., 12:9, 2020. DOI: https:// www.doi.org/10.1038/s41368-020-0075-9

Prati, C.; Pelliccioni., G. A.; Sambri, V.; Chersoni, S. \& Gandolfi, M. G. COVID-19: its impact on dental schools in Italy, clinical problems in endodontic therapy and general considerations. Int. Endod. J., 53(5):723-5, 2020.

Repici, A.; Maselli, R.; Colombo, M.; Gabbiadini, R.; Spadaccini, M.; Anderloni, A.; Carrara, S.; Fugazza, A.; Di Leo, M.; Galtieri, P. A.; et al. Coronavirus (COVID-19) outbreak: what the department of endoscopy should know. Gastrointest. Endosc., 2020. DOI: https://www.doi.org/10.1016/j.gie.2020.03.019

Sabino-Silva, R.; Jardim, A. C. G. \& Siqueira, W. L. Coronavirus COVID-19 impacts to dentistry and potential salivary diagnosis. Clin. Oral Investig., 24(4):1619-21, 2020.

Smith, A. C.; Thomas, E.; Snoswell, C. L.; Haydon, H.; Mehrotra, A.; Clemensen, J. et al. Telehealth for global emergencies: Implications for Coronavirus Disease 2019 (COVID-19). J. Telemed. Telecare, 2020. DOI: https://www.doi.org/10.1177/ 1357633X20916567

Sohrabi, C.; Alsafi, Z.; O'Neill, N.; Khan, M.; Kerwan, A.; Al-Jabir, A.; Iosifidis, C. \& Agha, R. World Health Organization declares global emergency: A review of the 2019 novel coronavirus (COVID-19). Int. J. Surg., 76:71-6, 2020.

Sun, P.; Lu, X.; Xu, C.; Sun, W. \& Pan, B. Understanding of COVID19 based on current evidence. J. Med. Virol., 2020. DOI: https:/ /www.doi.org/10.1002/jmv.25722

Wan, K. H.; Huang, S. S.; Young, A. L. \& Lam, D. S. C. Precautionary measures needed for ophthalmologists during pandemic of the Coronavirus Disease 2019 (COVID-19). Acta Ophthalmol., 98(3):221-2, 2020.

Wang, Y. Y.; Jin, Y. H.; Ren, X. Q.; Li, Y. R.; Zhang, X. C.; Zeng, X. T: wang, X. H. \& Zhongnan Hospital of Wuhan University No- vel Coronavirus Management and Research Team. Updating the diagnostic criteria of COVID-19 "suspected case" and "confirmed case" is necessary. Mil. Med. Res., 7(1):17, 2020.

Wang, X.; Pan, Z. \& Cheng, Z.S. Association between 2019-nCoV transmission and N95 respirator use. J. Hosp. Infect., 105(1):104-5, 2020.

Wu, F.; Zhao, S.; Yu, B.; Chen, Y. M.; Wang, W.; Song, Z. G.; Hu, Y.; Tao, Z. W.; Pei, Y. Y.; Yuan, M. L.; et al. A new coronavirus associated with human respiratory disease in China. Nature, 579:265-9, 2020.

Ye, Z.; Zhang, Y.; Wang. Y.; Huang, Z. \& Song, B. Chest CT manifestations of new coronavirus disease 2019 (COVID-19): a pictorial review. Eur. Radiol., 2020. DOI: https://www.doi.org/ 10.1007/ s00330-020-06801-0

Yu, Y. X.; Sun, L.; Yao, K.; Lou, X. T.; Liang, X.; Zhao, B. W.; Mu, Q. X.; Du, H.; Zhao, Y. \& Zhang, H. Consideration and prevention for the aerosol transmission of 2019 novel Coronavirus. Zhonghua Yan Ke Za Zhi, 56(0):E008, 2020.

Zhang, Z.; Liu, S.; Xiang, M.; Li, S.; Zhao, D.; Huang C. \& Chen, S. Protecting healthcare personnel from 2019-nCoV infection risks: lessons and suggestions. Front. Med., 14(2):229-31, 2020.

Dirección para correspondencia:

Britto Ebert Falcón-Guerrero

Av. Tarapacá \# 350.

Tacna

PERÚ

Email: artdent2000@hotmail.com

Recibido : 01-05-2020

Aceptado: 04-05-2020 